\title{
Free-Weight Resistance Training in Youth Athletes: A Narrative Review
}

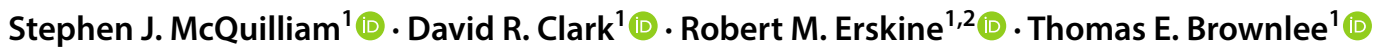

Published online: 23 June 2020

(c) The Author(s) 2020

\begin{abstract}
Generating high levels of muscular strength and power are important for success in sport and may have long-term implications for sporting careers in youth athletes. Importantly, maturation may confound the neuromuscular adaptations to resistance training when attempting to differentiate between training- vs. growth-induced strength and power gains; thus, potentially leading to erroneous conclusions regarding the efficacy of resistance training in youth athletes. The aim of this review was to critically appraise the literature concerning the efficacy of externally loaded free-weight resistance training on strength and power measures in youth athletes at different stages of maturity. Strength underpins power production; thus, developing strength through traditional resistance training methods can positively influence powerful sporting movements. In addition, weightlifting has the capacity to improve muscular power via explosive lower-body triple extension, which is essential for many sports. Despite the complexity of the techniques involved, it can be a safe and effective method to improve athletic qualities in young athletes, potentially more so than plyometric training. While low-load, high-velocity training can have a positive effect influence on high speed movements such as sprinting, the reduced intensity appears to be disadvantageous post peak-height velocity. Irrespective of age, well-coached progressive strength training adhering strictly to correct technique can then be periodised within a long-term athletic development program. It is important to primarily develop muscular strength, while concurrently refining the technical skill required for weightlifting. Physically mature athletes should undertake highintensity resistance training to maximise neuromuscular adaptations, leading to positive changes in strength and power.
\end{abstract}

\section{Key Points}

Irrespective of age, following an initial focus on fundamental movement techniques, strength development can be periodised within a long-term athlete program.

As strength fundamentally underpins power, it is important to first develop this, while concurrently refining the technical skills required for weightlifting.

Technically able physically mature athletes should undertake high-intensity resistance training ( $\geq 80 \%$ $1 \mathrm{RM}$ ) to maximise neuromuscular adaptations, leading to changes in strength and power.

Stephen J. McQuilliam

S.J.McQuilliam@2018.ljmu.ac.uk

1 School of Sport and Exercise Sciences, Liverpool John Moores University, Liverpool, UK

2 Institute of Sport, Exercise and Health, University College London, London, UK

\section{Introduction}

In many sports, the ability to generate high levels of muscular power is an important component for success [1]. Thus, practitioners aim to improve muscular power via effective and efficient training programs. Previously, a potentially misleading report based on the hospital admission records of injury cases concluded that resistance training (RT) was not safe in youth populations [2]. Further investigation determined that many of the recorded injuries were accidents resulting from incorrect exercise technique and/or poor supervision. More recent research has shown that RT in youth athletes can be a safe and beneficial training method $[3,4]$. Researchers have proposed that RT interventions in youth populations result in significant increases in strength, power and agility and reduced injury risk $[5,6]$. However, a point of conjecture surrounds RT best practice and the translation of this from the literature to the applied setting.

For young athletes, development of strength and power may have long-term implications for their sporting careers. The confounding factor of maturation on training adaptations in terms of training- vs. growth-induced strength/ power gains can complicate the training process. For 
example, chronologically older youth soccer players have been shown to be stronger in absolute terms but not when strength was normalised to body mass [7, 8]. Environmental factors, such as time restrictions due to sport-specific technical training and a limited number of appropriately qualified and experienced staff available to supervise and implement RT can also make translation of scientific literature into applied practice challenging [9].

The aim of this review was to critically analyse the scientific literature regarding the use and efficacy of RT regarding neuromuscular adaptations and how they translate into strength and power gains in youth sport. The objective is to provide recommendations based on the available evidence in the literature on the best practice regarding RT in youth sport, with particular reference to maturity status.

\section{Long-Term Athlete Development Models}

Practitioners and researchers have attempted to align various training methods with each stage of maturity in young athletes. As such, several training models have been proposed. Long-term athlete development (LTAD) models may aid in structuring a young athlete's training. An early wellknown LTAD framework was proposed by Côté [10], outlining three clear phases: sampling years (ages 6-12 years), specialising years (ages 13-15 years) and investment years (ages 16+ years). However, a potential problem is the classification of athletes based on the chronological age [11]. The chronological age of an individual is defined as a selected time point from date of birth [12]. In youth sport, chronological age is typically utilised to categorise age groups for competitions/academy squads [13]. However, individuals of the same chronological age can differ greatly in terms of biological age [14], which is defined as the stage of an individual's physical growth in relation to skeletal, sexual or somatic attributes [15]. The LTAD model by Bayli and Hamilton [16] attempted to correct for this by using biological age, through longitudinal monitoring of somatic variables. This allows practitioners to identify time periods of accelerated growth, regarding peak-height velocity (PHV, the phase where peak rate of skeletal growth occurs) and peak-weight velocity (PWV, the phase where peak rate of maturationassociated skeletal muscle accretion occurs) for a specific athlete and programme training accordingly [16]. The timing and speed of biological maturation is highly individual and, therefore, it is an important factor to consider when designing a training program, as it has been proposed to be more appropriate than chronological age [17].

The LTAD model suggested by Bayli and Hamilton [16] has been structured utilising supposed "windows of opportunity", during which certain physiological characteristics are theoretically more responsive to training stimuli. However, this theory lacks evidence due to the diversity and range of components that contribute to a variety of sports [11]. In contrast, the Youth Physical Development (YPD) model suggests that most physical qualities are trainable throughout maturation, with different mechanisms underpinning adaptations [17]. A meta-analysis revealed that youth athletes may benefit from RT to the same extent, independently of age [6], although a later review suggested that absolute increases in strength were greater during or after PHV than those seen pre-PHV [18]. Owing to dissimilar reports, the results should be interpreted with caution. These models provide a guideline for effective training prescription; however, differences in training history, biological age and sporting requirements will influence the implementation and resulting adaptations.

\section{Influence of Maturation on Strength, Power and Speed}

Maturity status has been identified as a contributing factor to a variety of different physical performance indicators, such as strength, peak power, sprinting, change of direction speed (COD), as well as both anaerobic and aerobic performance $[19,20]$. It is important to track biological maturation longitudinally, as those who mature early generally have an advantage over their late-maturing peers due to greater strength and power at that point in time. In addition, there may be a disproportionate number of young athletes with birth dates in the first quarter of the selection year due to an advanced maturity status, known as the relative age effect [21]. Although individuals may be physically dominant during adolescence due to advanced maturation, this may not continue to be the case when fully mature [13]. During adolescence, it is possible to be at very different stages of biological maturation with the same chronological age, thus practitioners need to be aware that individuals within the same cohort may require different training stimuli.

The biological changes that occur from childhood through to full maturity directly influence strength and power via multiple mechanisms. Prior to PHV, increases in strength and power via training are suggested to be a result of improved neuromuscular activation [11, 19, 22, 23]. During this stage of maturation (pre-PHV), relatively low concentrations of circulating androgens, such as testosterone and growth hormone, limit the capacity for skeletal muscle morphological adaptations [24]. A significant phase of growth starts in girls and boys between 9-12 and 12-14 years old, respectively. In relation to biological maturation, this equates to approximately 1.5 years prior to PHV [11]. This period of elevated growth rate lasts until 0.5-1 year post-PHV [19], 
during which time another large increase in muscular power occurs [11]. This increase in strength occurs in both sexes, but more so in boys due to more testosterone being secreted by the testes than the ovaries [25]. As testosterone is a potent stimulator of muscle protein synthesis [26] and inhibitor of muscle protein breakdown [27], it follows that, during this period, boys more than girls experience a significant accretion of muscle mass, which is the main physiological determinant of maximum strength [28]. Muscular strength is the ability to apply force on an external object. As the ability to generate force improves, more force can be applied in the same time frame, resulting in greater power production. As such, similar physiological mechanisms may drive maturation-associated increases in both attributes [29]. During adolescence, males exhibit a re-distribution of muscle fibre type from predominantly type I to type II fibres [30, 31]. Type II fibres have a greater cross-sectional area (CSA), allowing for greater force production than type I fibres as well as a faster cross-bridge attachment/de-attachment cycle allowing for a faster shortening velocity, thus greater power production [32]. In addition, an increase in limb length during skeletal growth may simultaneously increase the internal moment arm, thus increasing torque production [23].

Although there are large increases in strength and power during PHV, the greatest gains tend to occur at onset of PWV. This is typically between 6 months to a year after PHV, when the rate of lean mass accruement is greatest [33]. In relation to muscle morphology, three key factors influence power generation: muscle physiological CSA, muscle fascicle length and muscle fascicle pennation angle [34], all of which can be assessed non-invasively via ultrasonography. Briefly, muscle physiological CSA represents the CSA of the total number of muscle fibres, perpendicular to their long axis [35]. Muscle fascicle length is defined by the number of sarcomeres in series, with longer muscle fibres able to contract faster than shorter fibres. Muscle fascicle pennation angle, i.e. the angle at which fascicles insert into the aponeurosis, increases due to fibre hypertrophy, which is caused by an increase in the number of sarcomeres arranged in parallel [34]. Muscles with larger fibre CSAs and greater pennation angles produce greater forces, while muscles with longer fascicles and smaller pennation angles have a greater shortening velocity $[34,36]$. Interestingly, muscle fascicle length and pennation angle appear to be independent parameters of maturation. There appears to be no difference in muscle fascicle length when normalised to body height and muscle fascicle pennation angle seems to be comparable between children, adolescents and adults [37-39]. However, it should be noted that 2D ultrasound imaging of muscle architecture may not accurately quantify differences in a 3D structure, particularly if extrapolation of fascicle measurements is necessary due to limitations with transducer width [40]. Further, there are limited data regarding natural development of the aforementioned physical attributes in terms of longitudinal studies and the impact physical training may have.

To contextualise the above points, increases in strength in the absence of gains in body mass have a greater impact on sports where athletes propel their own body mass, for example sprinting and jumping. Alternatively, increases in strength with gains in body mass which are seen during PWV, may have greater influences where both high-force movements and momentum become important in sports such as rugby when tackling and breaking tackles. Realistically, practitioners should expect increases in absolute strength as a consequence of lean accretion with maturation, while increases in strength normalised to body mass are more likely the product of specific exercise training [7].

\subsection{Response to Training}

There are no minimum age guidelines for youth participation in RT. National governing bodies for strength and conditioning support RT for children when the child is both physically and mentally prepared to engage in sport $[3,41]$. This is determined based on their ability to follow instruction, which is central in ensuring safety [41].

As previously mentioned, adaptations differ according to maturity status in youth cohorts [11]. Load-velocity profiles can estimate maximal force, peak power and velocity capabilities in the assessed movement. Meylan et al. [42] reported different force-velocity-power (kinetic) responses between biological age groups. Following an 8-week RT intervention pre- and mid-PHV cohorts experienced increases in maximal velocity, which in turn improved maximum power on a machine-squat, whereas the post-PHV group expressed increases in power via improved maximal force and velocity. Benefits were more pronounced in the post-PHV group, particularly where high levels of force and power were required, such as 1RM strength test and $10 \mathrm{~m}$ acceleration. Furthermore, Rodríguez-Rosell et al. [43] implemented a low-load, high-velocity RT intervention, applying the same duration and frequency in youth soccer players. All groups (U13, U15 and U17) showed significant improvements in strength, jump and sprint assessments, although the degree of improvement diminished with increasing chronological age. The authors concluded that mature athletes require greater relative training loads to maximise adaptations, based on the higher relative maximal strength or 1RM [43].

The training status of those included in interventions and subsequent reviews is an important factor as this may influence efficacy of an intervention. In a systematic review and meta-analysis by Behm et al. [44], data indicated that untrained youth experience larger increases in both jump and sprint assessments than their trained counterparts due to inferior baseline results and RT being an unfamiliar stimulus. Behm et al. [44] proposed that trained youth might adapt through 
neural and morphological adaptations whereas untrained participants will improve primarily via neural pathways. Untrained youth may encounter a greater learning effect due to their relative inexperience. Therefore, untrained subjects may have to initially improve their motor-unit recruitment before morphological changes can be observed [45]. However, a subsequent review by Slimani et al. [46] reported no significant effect of training status on improvements in squat jump (SJ) performance. Slimani et al. [46] attributed the variances in findings to differences in methodology, as they focused on vertical jump performance exclusively, whereas Behm et al. [44] included other parameters, such as strength and sprint speed. When considering this potential greater trainability, methods derived from research in untrained youth populations should be implemented in high-level (trained) youth athlete settings with caution.

Despite a plethora of research examining outcome measures i.e. vertical jump and sprint performance, the training history of the participants involved is key. In addition, there appears to be limited research on the underpinning mechanisms behind these physiological adaptations to RT in adolescent athletes [47]; thus further investigation is required.

\section{Resistance Training Methods}

The term "resistance training" is an all-encompassing term used throughout the literature referring to a variety of methods, primarily machines and/or free-weights. These methods have the capacity to augment both muscle physiological CSA and neural activation, which influence muscle strength and power. This section will focus on interventions primarily utilising free-weight RT as well as the commonly used Smith machine. Although the Smith machine it is not a free-weight exercise, it has a prominent place in strength-training research [48]. A number of freeweight training methods can induce positive adaptations in strength and power in youth cohorts, including heavy strength training, weightlifting (WL), peak-power training and a combination of these [49-51]. However, different modalities appear to be more beneficial depending on physical characteristics targeted and stage of maturity. Free-weight training refers to a load that moves freely in space, e.g. the barbell back squat and that is not attached to another support structure. Free-weight RT is seen as a more efficient method of improving strength. This allows for large compound movements coupled with reduced stability; therefore, increasing the recruitment of stabilising musculature around the primary muscles as well as superior reproduction of sporting actions, such as vertical jumping [6]. The following sections will aim to examine various free-weight RT modalities in youth populations and the influence on strength and power.

\subsection{Strength Training}

Maximal strength underpins athletic muscular performance qualities, such as peak power, by increasing maximal force potential [52]. A significant correlation exists between higher relative training intensities (\%RM) and improvements in maximal strength and motor skill performance in youth populations [45]. High levels of strength may influence sport-specific skills and increase jump height and sprint performance $[53,54]$. Strength training can be defined as high-load RT relative to an individual's maximal strength ( $\geq 80 \% 1 \mathrm{RM})$, utilising two to four sets at low-repetition ranges $(\leq 6)$ [55]. Furthermore, a recent meta-analysis highlighted that the most effective intensity to improve strength in youth athletes is $80-89 \%$ 1RM [6]. Training at high percentages of maximal strength has an important role in changing tendon properties in adolescent athletes [37]. Moreover, reduced tendon strain via an RT-induced increase in tendon CSA may reduce occurrence of tendinopathies [56]. High-intensity RT appears to be a fundamental component of a training regime in order to prepare a young athlete for sports participation via increased proxies of performance and reduced risk of injury.

As mentioned previously, improving an athlete's strength may increase both initial acceleration and maximal sprint speed. Improving initial acceleration may be highly beneficial in sports such as soccer with approximately 60 accelerations occurring per match [57]. Impulse (the product of force multiplied by time) is a key determinant of acceleration. However, as time is restricted during the ground contact phase, maximising force production within this time window is vital [58]. There is a strong correlation between absolute squat strength and sprint performance due to an associated greater rate of force development (RFD) and in turn, ground reaction force $[59,60]$. Thus, maximal strength has been identified as an important factor to maximise initial acceleration, where ground contact times $>200$ ms enable greater force transfer [59]. These measures require the recruitment of the lower body musculature in one coordinated movement; consequently, the squat has become the cornerstone of many strength-training programs [51,61].

When programmed appropriately, adolescents respond positively to high-intensity RT (> 80\% 1RM). Keiner et al. [62] compared front and back squat strength in adolescent soccer players and weightlifters. As expected, weightlifters outperformed soccer players at all age groups. Notably, in the 17-19 years age group, youth weightlifters demonstrated $2.1 \pm 0.1 \times$ bodyweight $5 \mathrm{RM}$ back squat in comparison to the soccer players who produced a $1.3 \pm 0.2 \times$ bodyweight $4 \mathrm{RM}$. Importantly, the weightlifting group completed the test to full-depth and for an extra repetition, whereas the soccer players were limited to parallel depth (thighs parallel to the 
floor). The full-squat results in a lower load as compared to the parallel squat, meaning that differences in strength between the two cohorts may be greater than reported [63]. These studies highlight the efficacy of high-intensity RT in youth populations.

\subsubsection{Volume and Intensity}

Training programs consist of numerous variables, more than just exercise selection. When designing a RT program two key components are primarily considered: training volume and training intensity. There are multiple ways to calculate both components. Volume may be quantified via a repetition, volume load and volume index method and intensity as percentage of 1RM or average load for an exercise/overall session, for instance [64]. In a sport setting the demands of competition and the time available may influence these variables, meaning time efficient training methods are of great value. When working with youth athletes, biological maturity and phases of accelerated growth must also be considered as part of LTAD [65].

\subsubsection{Intensity}

When the training aim is to build strength in youth athletes high-intensity RT ( $>80 \%$ 1RM) has been suggested to be the most effective method and benefits can become apparent within a small timeframe [6]. Chelly et al. [51] implemented an 8 -week, twice-weekly squat training program, comprising three sets at intensities between 80 and 90\% 1RM in U18 soccer players with no RT experience. These loads were chosen because they are suggested to increase RFD, particularly in weaker/untrained participants [29, 52]. Along with a low-RT volume, there were significant increases in peak power, $40 \mathrm{~m}$ sprint, $\mathrm{SJ}$ and repeat bounding performance with no increases in thigh CSA; thus the adaptations were suggested to be neurological. Despite improvements in SJ, there were no significant improvements in countermovement jump (CMJ). Speirs et al. [66] reported similar results after a 5-week intervention, utilising 75-92\% 1RM in U19 rugby union players with at least one year of RT experience. Although shorter in duration and a similar weekly RT volume to Chelly et al. [51], there were significant increases in back squat 1RM, $40 \mathrm{~m}$ sprint time and agility performance. In combination, there is evidence to suggest high-intensity RT with low-training volume can have an impact on acceleration, jump and COD in a short time period (Table 1), especially if the stimulus is unfamiliar.

\subsubsection{Volume}

Alongside training intensity, the volume of training must be considered in the athletic development of youth athletes.
Both Hammami et al. [67] and Styles et al. [68] implemented twice-weekly high-intensity RT interventions in adolescent athletes over 8 and 6 weeks respectively. Both studies reported significant improvements in strength and $20 \mathrm{~m}$ sprint time. The largest improvements in the study by Hammami et al. [67] occurred in $5 \mathrm{~m}(11.1 \%)$ and $10 \mathrm{~m}(9.4 \%)$ sprint tests, which is in line with the previous research [54]. Furthermore, the largest improvements in agility were observed in tests that required a greater number of direction changes. The greater number of accelerations and decelerations associated with multiple direction changes allow for more instances, where greater strength could influence the test outcome. Hammami et al. [67] also reported significant increases in both SJ and CMJ. This is in contrast to the previous research where only increases in SJ were seen [51]. Although both interventions incorporated high-intensity loads between 70 and $90 \% 1 \mathrm{RM}$, the main difference in protocol was training volume. Whereas Chelly et al. [51] implemented eight sets per week totalling 18 repetitions, Hammami et al. [67] completed 42 sets, totalling 186 repetitions. Training volume has been highlighted as an important stimulus for adaptation in athletic populations [69]. However, despite a far lower training volume than Hammami et al. [67], Chelly et al. [51] also produced significant increases in 1RM half-squat strength, squat jump, as well as $5 \mathrm{~m}$ and $40 \mathrm{~m}$ sprint performance in the same population. Irrespective of volume, both Chelly et al. [51] and Hammami et al. [67] attributed the improvements to neural adaptations, with neither study finding significant changes in thigh CSA, which is in line with a previous review by Ford et al. [11]. A multitude of factors can influence adaptation including previous RT experience, biological age as well as training volume and intensity $[6,18,44]$ but it appears that higher training intensities ( $>80 \% 1 \mathrm{RM})$ and low volume can lead to similar increases in performance as low-intensity, high volume (Table 1).

\subsubsection{Long-Term Training}

The previously mentioned studies were all short in duration (e.g. $\leq 8$ weeks) in chronologically older youth athletes, where biological growth might not be significant enough to affect the RT-induced changes in strength or power. Sander et al. [70] conducted a 2-year intervention in young soccer players to observe the influence of regular RT alongside soccer training on strength and sprint performance. At the start of the intervention, groups consisted of players from U13, U15 and U17 squads matched with control groups performing only soccer training. Key low-body exercises included both the front and back squat, as well as the deadlift, all at an intensity of 75 to $90 \% 1 \mathrm{RM}$. The largest effect size was seen in the U13s for both squat tests (back squat $\mathrm{ES}=2.0$, 


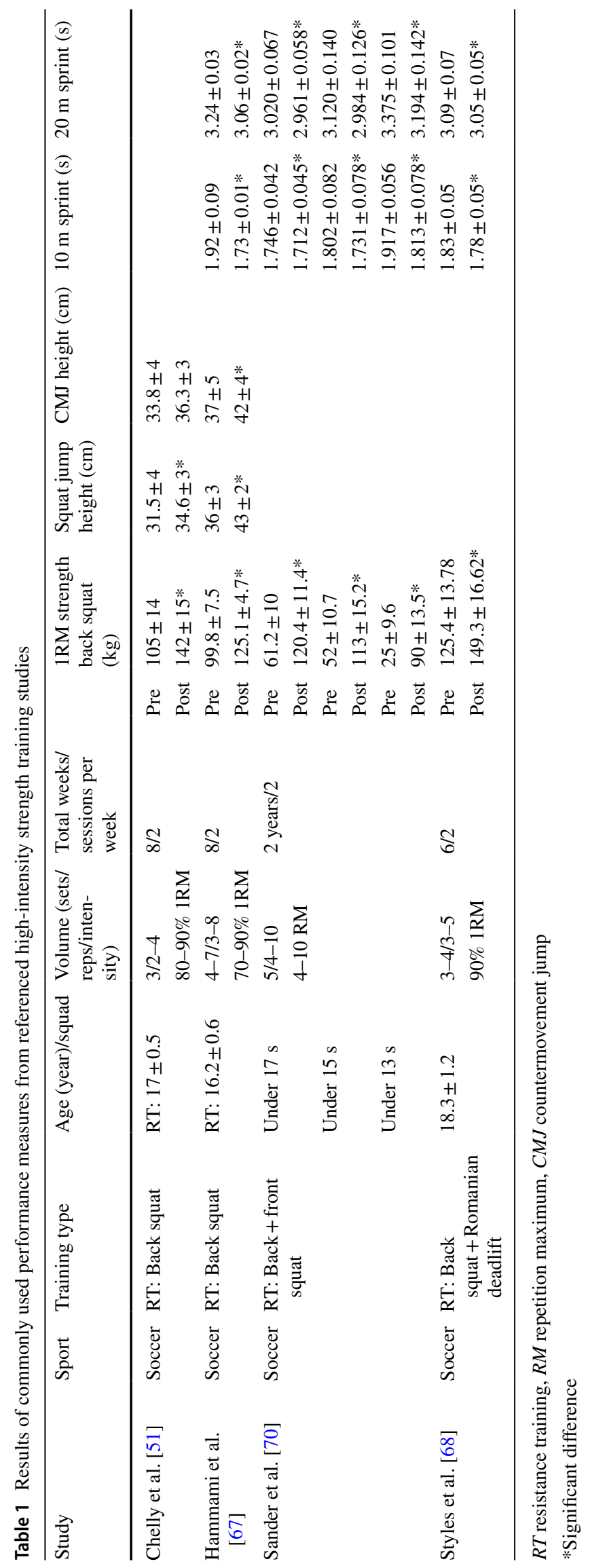


front squat $E S=1.9)$. This is supported by Lesinski et al. [6], where larger effect sizes were seen in younger ( $\leq 13$ years) than adolescent males (14-18 years) $(\mathrm{ES}=1.35$ vs. 0.91$)$. Strength training also significantly improved $30 \mathrm{~m}$ sprint performance at all $5 \mathrm{~m}$ intervals when compared with the control group in both the U13s and U15s squads. The U17s failed to improve $10 \mathrm{~m}$ sprint performance, which may have been due to more variability in the percentage change [70]. Of the control groups, the U13s and U15s improved 15-30 m and $10-30 \mathrm{~m}$ sprint interval performance, thus suggesting maturation and/or soccer training influenced these variables. However, the U17s control group did not improve any sprint times. Because this group would not be expected to demonstrate significant maturation-related growth, these results suggest that soccer-specific training does not improve sprint performance and that the improvements in the U13 and U15 control groups were due to maturation-related growth, not soccer-specific training [70]. However, maturity status was not assessed and therefore could not be used a covariate in subsequent analysis to delineate the RT effect from the soccer training effect. Nonetheless, Sander et al. [70] highlights that long-term RT in adolescent athletes is effective in improving strength and in turn sprint performance in youth athletes from 5 to $30 \mathrm{~m}$.

When youth athletes are systematically exposed to high-intensity RT over a prolonged period of time (e.g. $\geq 12$ months) there can be significant increases in maximum strength as well as sport-specific assessments, such as vertical jumps and sprinting performance (Table 1). The literature suggests that benefits can be seen at all stages of biological maturity; therefore, high-intensity RT should be included throughout an athlete's development.

\subsection{Weightlifting}

As previously stated, high levels of muscular power are important for sports performance [1]. Training to increase maximum strength augments the capacity to develop power [71]. A holistic training program that incorporates maximum strength and WL variations can facilitate this transfer [72]. In WL movements, the emphasis is typically on movement speed, at moderate to heavy loads. As a result, WL can increase motor-unit recruitment and therefore RFD [73]. The two primary WL lifts are the clean and jerk and the snatch, with derivatives, such as the hang-power clean involving high force and velocity outputs [72], which are the components of power. Mechanically, WL movements align with the principle of specificity by replicating kinematic and kinetic characteristics of the vertical jump [74]. In contrast to traditional RT methods, there is limited research on WL in youth populations.

Despite limited research in the area, results appear to be promising at each stage of biological maturity. Chaouachi et al. [50] compared WL to traditional RT and plyometric training (PLYO) in 10- to 12 year-old males for a period of 12 weeks. The RT intervention utilised squats and lunges, whereas the WL program implemented clean and snatch variants. Both groups followed identical set and repetition schemes in an attempt to equalise training volume (i.e. 1-3 sets $\times 8-12$ reps). The results showed no clear differences between RT and WL in terms of increases in $5 \mathrm{~m}$ acceleration, $20 \mathrm{~m}$ flying sprint or vertical CMJ performance but there was a likely benefit for WL in horizontal CMJ distance. Importantly, there were larger effect sizes for the WL group when compared with the PLYO group for changes in all strength and power variables. Subjects had no previous RT experience and the concentric phase of the squat was not explosive which should be considered when interpreting these results. In an adolescent cohort with limited RT experience, Channell, Barfield [75] compared the effect of WL vs. traditional RT on vertical CMJ performance. Each intervention group shared a number of common exercises, while completing three group-specific core lifts. Similar to Chaouachi et al. [50], after 8-week, both groups saw similar improvements in vertical CMJ performance. Taken together, the results suggest that WL may be more effective when improving muscular power and subsequent athletic tasks than PLYO in young populations.

Following a period of WL, Channell and Barfield [75] suggested improvements in strength and power seen were likely due to neural adaptations, i.e. greater neuromuscular voluntary activation of the agonists, synergists and stabilisers, all contributing to improve technique, as well as muscular force and contraction velocity. Arabatzi and Kellis [76] examined differences in EMG activity between WL and traditional RT to explain why WL may produce better jump performance. They implemented an 8-week highintensity (80-90\% 1RM) intervention in resistance-trained male students, comparing WL variants to traditional RT. They concluded that greater improvements in SJ, CMJ and drop jump (DJ) with WL were due to increased agonist muscle (rectus femoris) activation, reduced antagonist muscle (biceps femoris) co-activation and an increased leg stiffness. The RT group also showed an increased leg stiffness, seen as a decreased change in the body's centre of mass during the eccentric phase of a drop jump test. However, there was an increased activation of both agonist and antagonist muscle groups. In powerful actions, such as jumping, increased antagonist muscle co-activation may reduce velocity towards the end of the movement, limiting power production [77]. The results of Arabatzi and Kellis [76] highlight that ballistic RT in the form of WL may produce a more beneficial neural activation pattern of agonist and antagonist muscles that is not prevalent with traditional RT. Thus, incorporating WL into a training program appears to be important for improving ballistic actions. 
Weightlifting is a training method that has the capacity to improve muscular power by utilising the explosive lower body triple extension, which is essential for sprinting and jump variants in many sports [1]. Despite the complexity of WL, it can be an effective method to improve athletic qualities in young athletes at each stage of biological maturity (Table 2), with minimal injury risk when appropriately supervised [50].

\subsection{Peak-Power Training}

Training methods focusing on low-load and high-velocity movements are suggested to be beneficial for sprinting as well as vertical and horizontal jump performance, particularly in pre-PHV athletes [78]. Much like WL, training of this nature centres on peak-power production, which can occur at different intensities according to the exercise. Cormie et al. [79] reported peak power with external loads occurred at $0 \%, 54 \%$ and $80 \%$ of $1 \mathrm{RM}$ jump squat, back squat and power clean, respectively. However, always training at peak power may limit further improvements, as strength would remain underdeveloped [29]. This is important, as greater levels of strength relative to body mass correlate strongly to CMJ height [80].

This method has been suggested to improve strength and power via neural mechanisms, making it ideal for pre-PHV athletes, when morphological adaptations are limited. However, post-PHV, increases in peak power is predominately via an increase in force generation [42]. Thus, training at peak power would result in a sub-optimal training load. In a long-term study, Gonzalez-Badillo et al. [81] implemented a twice-weekly, high-velocity RT intervention in Spanish academy soccer players, where they utilised low volumes of squats at loads of 45-59\% $1 \mathrm{RM}$ combined with jump and sprint training. Interestingly, after 6 months of RT, both the U16 and U18 groups matched or outperformed the U21 control group in the isoinertial squat strength test, vertical CMJ and $20 \mathrm{~m}$ sprint performance tests. Thus, it could be argued that 6 months of RT produced similar or greater gains than 5 years of soccer training and maturation combined. However, a limiting factor is that there were no measures of maturity or age-matched control groups, as maturation will have likely influenced these results. Rodríguez-Rosell et al. [43] employed a similar design but incorporated age matched controls, together with U13, U15 and U17 male soccer players who had no prior RT experience. However, the intervention was much shorter in duration. Over 6 weeks, a combination of high-velocity full squats (45-60\% 1RM), jumps, sprints and COD drills significantly improved iso-inertial squat load at $1.00 \mathrm{~m} \cdot \mathrm{s}^{-1}(\sim 56 \% 1 \mathrm{RM})$, vertical CMJ and $20 \mathrm{~m}$ sprint performance in all groups, with the percentage increase reducing with advancing

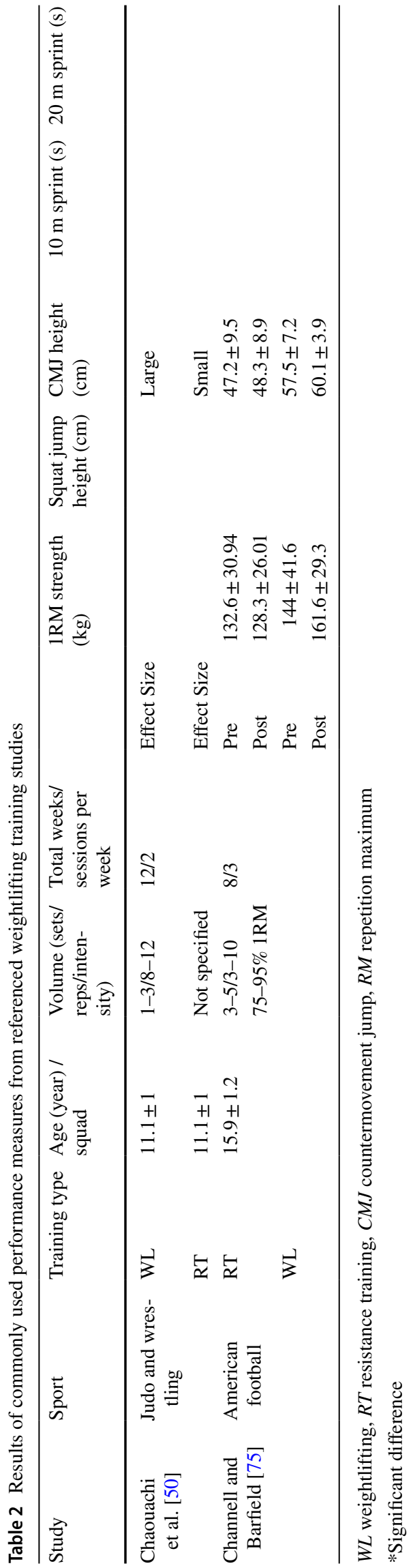


chronological age. Whereas the U13s and U15s were significantly better than their age-matched control group in all measures post training, the U17s outperformed their control group in the sprint and strength assessments only (Table 3). The results presented here can be explained by the different kinetic responses that increase peak power at different stages of biological maturity [42]. While pre- and mid-PHV individuals appear to increase peak power primarily via increasing movement velocity, post-PHV individuals appear to do this principally by increasing force output [42]. Although there were increases in strength in the U17 age group the different kinetic responses reported by Meylan et al. [42] suggest that low-load, high-velocity training is sub-optimal at the later stages of biological age. In addition, benefits from this method are potentially due to limited/no-previous RT experience; thus, it is less likely to have an effect with increased training age. This suggests that low-load, high-velocity training may be more beneficial for younger than for more mature athletes. For physically mature athletes, however, the incorporation of high-intensity strength training is likely required to elicit greater improvements in performance.

\subsection{Combined Methods Resistance Training}

As part of a holistic RT program, it is unrealistic to implement one method in isolation as seen in certain studies [50, $51,81]$. Aspects of the force-velocity curve are involved in many sporting actions. The synergistic benefits of a combined-method approach on improvements in sprint performance have previously been acknowledged. In a systematic review and meta-analysis, the largest effect sizes on sprint performance were seen when back squat, loaded SJ/CMJ and PLYO were implemented concurrently $(E S=-1.20)$ [60]. The effect was much greater as compared to back squat $(\mathrm{ES}=-0.81)$ and loaded jump training alone $(\mathrm{ES}=-0.29)$. A similar pattern for training intensity became apparent with a combination of high ( $>85 \%$ of $1 \mathrm{RM})$ and very light $(<40 \% 1 \mathrm{RM})$ loads producing the largest effect size $(\mathrm{ES}=-0.82)$. However, lower effect sizes where seen when high $(\mathrm{ES}=-0.52)$ or low $(40-59.9 \% 1 \mathrm{RM}, \mathrm{ES}=-0.16)$ loads were used in isolation. Interestingly, medium loads (60-84.9\% 1RM, ES $=-0.97)$ in isolation produced the largest ES. With regards to volume, there was a moderate relationship with greater training frequency and sprint performance $(r=0.50 ; p=0.001)$ and longer rest intervals $(r=-0.47 ; p \leq 0.001)$ but no correlations for the number of exercises, sets or repetitions per set. Seitz et al. [60] suggested that a combination of high-, medium- and very light loads was the most effective approach to improve sprint performance. Although the participants in the review by Seitz et al. [60] (13-25 years of age) did not include those prePHV and continue into adulthood, it is important to note that

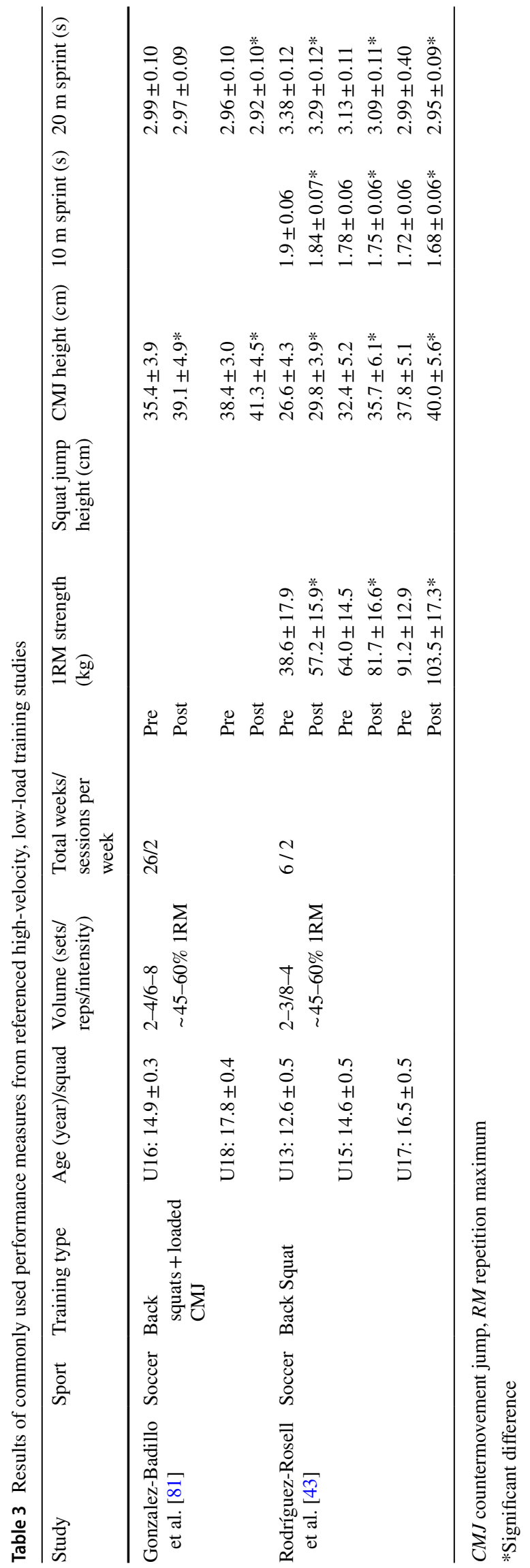


Seitz et al. [60] reported a non-significant correlation for both age and height regarding the effect of RT on sprint performance. This demonstrates that a variety of external loads and, therefore, velocities are beneficial at all age groups.

Recently, the combination of both high- and low-load RT within a single training session has gained popularity due to its time efficiency, often referred to as complex training (CT) [82]. A systematic review found CT to be significantly more effective at improving $20 \mathrm{~m}$ sprint times and 1RM strength as compared to other RT methods, but acknowledged that single study outliers may have influenced this [83]. Although no significant differences were reported concerning changes in jump, $5 \mathrm{~m}, 10 \mathrm{~m}, 30 \mathrm{~m}$ and $40 \mathrm{~m}$ sprint performance between $\mathrm{CT}$ and other methods, the improvements were associated with lower training volumes. In addition, the authors proposed that the potentially novel exposure to highintensity RT might have been responsible improvements following CT, as exposure to high-velocity movements would occur as a result of sport-specific training. Therefore, youth athletes may benefit from training at a range of intensities as part of their LTAD.

In youth cohorts, it is necessary to consider the influence of maturity status on the effectiveness of CT due to the different mechanisms responsible for training adaptations at each stage of maturity [11]. Both Lloyd et al. [49] and Radnor et al. [84] examined the adaptation to traditional RT, PLYO and CT in untrained pre- and post-PHV groups. In both short-term interventions, PLYO was a more effective training method to improve jump and sprint assessments in the pre-PHV groups, while CT was more effective at postPHV [49, 84]. Radnor et al. [84] continued to specify that $\mathrm{CT}$ and RT were more effective for improving variables that required high concentric force production, such as initial acceleration, whereas PLYO was more effective at improving peak running velocity and reactive strength index [84]. Although the RT protocol differs from the suggestions of Lesinski et al. [6], the results support the incorporation of RT, particularly post-PHV, where greater increases in maximal force and thus peak power are possible [42].

To the authors' knowledge, only one long-term study has examined WL as part of a CT program in youth athletes. Pichardo et al. [85] examined the effect of incorporating WL into a RT program on isometric mid-thigh pull (IMTP), vertical CMJ, horizontal $\mathrm{CMJ}$ and $30 \mathrm{~m}$ sprint performance. The original RT program (CRT) already comprised traditional RT and plyometric movements (CRT). In the WLRT group, conventional exercises, such as the deadlift and a plyometric movement were replaced with WL exercises for 28 weeks (WLRT), split into initial light-load technique training followed by 14 weeks at higher training intensities. Both groups significantly improved IMTP, vertical CMJ, horizontal CMJ and sprint performance (10-30 m) to a similar extent post training. However, the timing of these changes varied between groups, with both groups increasing absolute IMTP force mid-way through the training period, but only the CRT group displayed improvements in both $20 \mathrm{~m}$ and $30 \mathrm{~m}$ sprint tests. From mid-way, training intensity increased in both groups and from mid- to post-training, there were greater percentage improvements in all jump and sprint measures as compared to pre- to mid-training, particularly in the WLRT group. The authors proposed that the increased intensity aided the transfer of improved strength into improved power. The study was not without its limitations, namely the absence of a control group and the inability to distinguish between the effects of training $v s$. biological maturation due to no measure of maturity. However, with this study, Pichardo et al. [85] demonstrated the importance of developing strength prior to power, and that longitudinal studies are required to demonstrate the translation of strength into power.

The results from Lloyd et al. [49], Radnor et al. [84] and Pichardo et al. [85] highlight the importance of incorporating both high-force and high-velocity training to improve a range of strength and power measures (Table 4). Improving maximum force production becomes increasingly important post-PHV, due to the maturation-associated muscle growth allowing greater force outputs to improve peak power [11, 42]. When incorporated alongside loaded ballistic exercises, research would suggest there are greater improvements in powerful dynamic sporting actions post training, providing strength has already been developed.

\section{Conclusion}

The aim of this review was to critically appraise the scientific literature regarding the use and efficacy of RT in youth sport. Resistance training is an important aspect of a young athlete's development and is a safe and effective method when appropriately planned and supervised [3]. Based on the available evidence in the literature, the objective of this review was to provide recommendations on best practice regarding intensity and volume of RT/WL in youth athletes with specific reference to maturity status, which we have highlighted during the review and summarised below.

\subsection{Practical Implications and Future Research}

Irrespective of age, following an initial focus on fundamental movement techniques, such as the squat and hip-hinge, strength development can then be periodised within a LTAD program. As strength fundamentally underpins power, it is important to first develop this, while concurrently refining the technical skill required for WL. Physically mature athletes should undertake high-intensity RT to maximise 


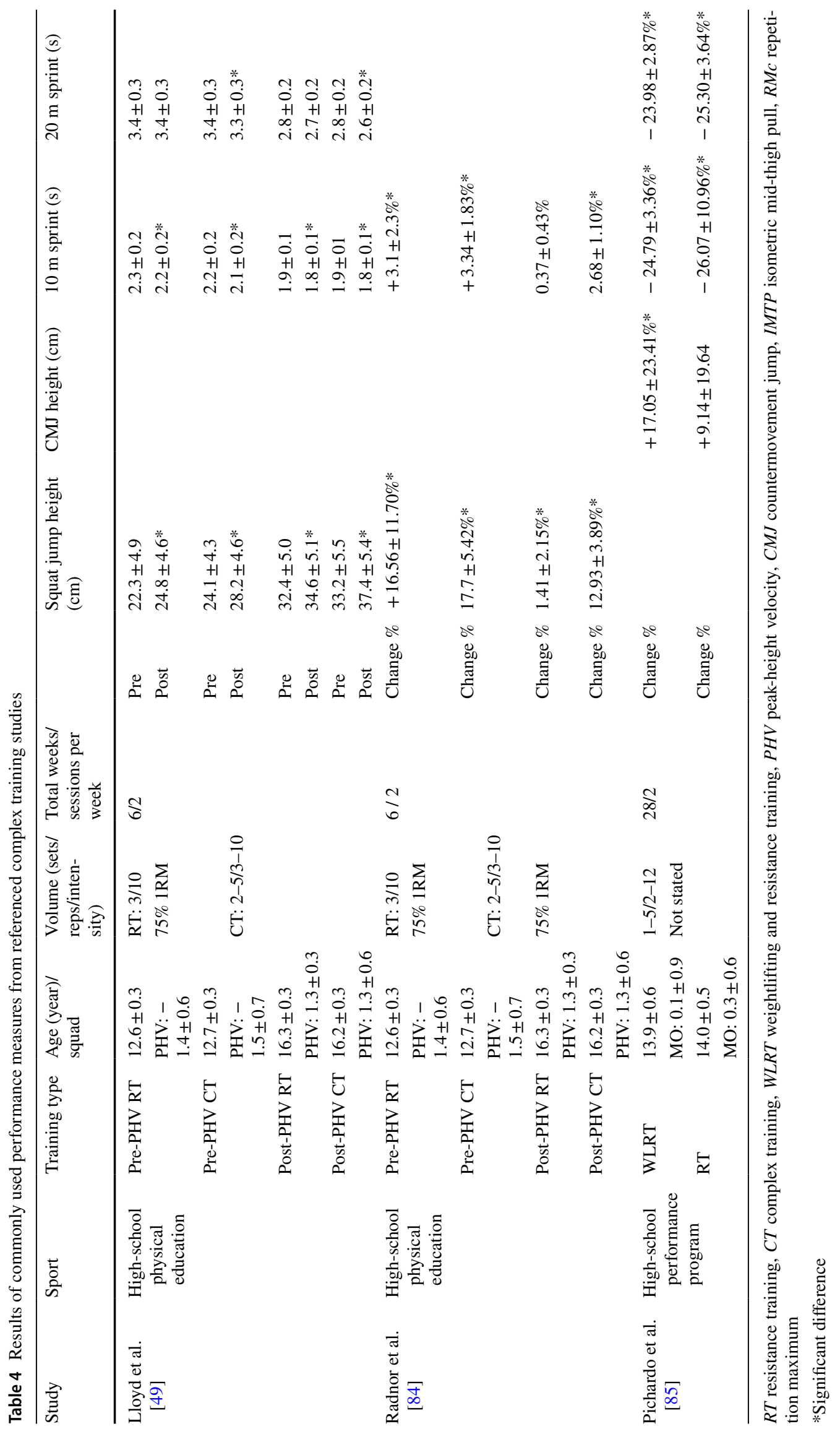




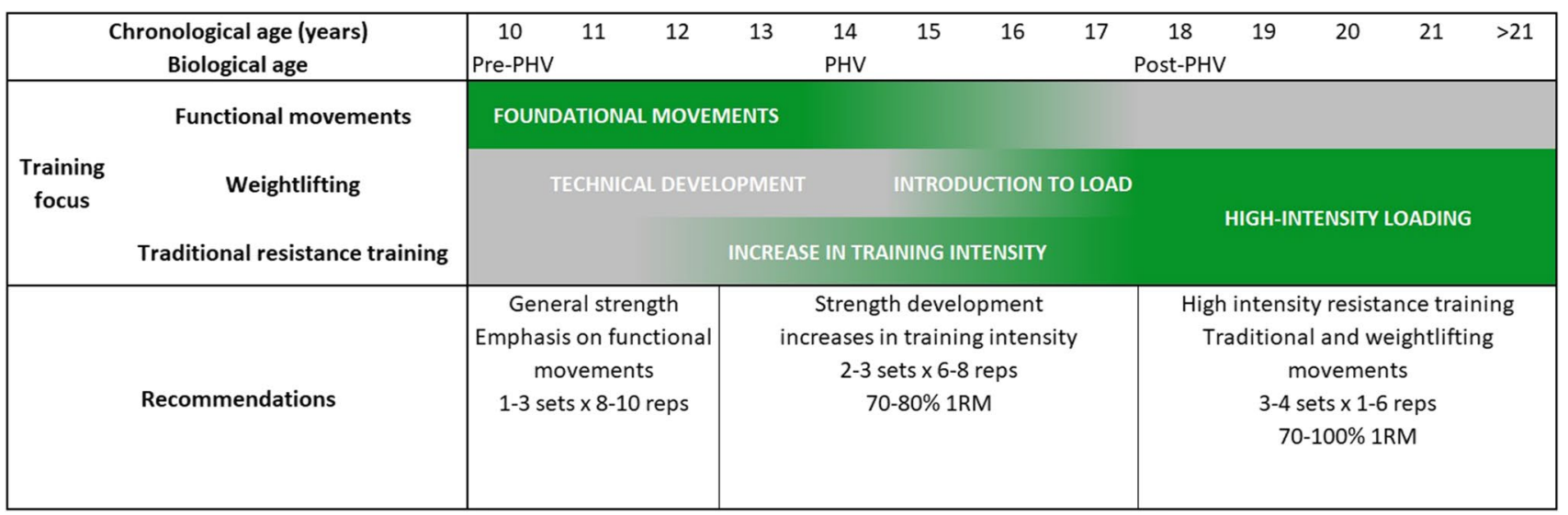

Fig. 1 Evidenced-based recommendations for developing strength and power within a holistic long-term athletic development plan in youth athletes. Grey refers a lower focus, green refers to a greater training focus. $P H V$ Peak-height velocity, reps repetitions, $R M$ repetition maximum

neuromuscular adaptations to RT, leading to changes in physical performance (Fig. 1).

It is important to consider that RT is a component of a larger sport-specific training structure, where time availability for RT/WL may be limited. Thus, future research should investigate various low-volume, high-intensity RT/ WL training methods to determine the required volume of training to elicit improvements in physical performance and physiological adaptations in youth athletes.

Author contributions SM drafted the manuscript. TB, DC and RE edited and revised the manuscript. All authors approved the final version before submission.

Data Availability Statement Data sharing was not applicable to this article as no datasets were generated or analysed during the current study.

\section{Compliance with Ethical Standards}

Funding No sources of funding were used to assist in the preparation of this article.

Conflict of interest Stephen McQuilliam, David Clark, Robert Erskine and Thomas Brownlee declare that they have no conflicts of interest relevant to the content of this review.

Open Access This article is licensed under a Creative Commons Attribution 4.0 International License, which permits use, sharing, adaptation, distribution and reproduction in any medium or format, as long as you give appropriate credit to the original author(s) and the source, provide a link to the Creative Commons licence, and indicate if changes were made. The images or other third party material in this article are included in the article's Creative Commons licence, unless indicated otherwise in a credit line to the material. If material is not included in the article's Creative Commons licence and your intended use is not permitted by statutory regulation or exceeds the permitted use, you will need to obtain permission directly from the copyright holder. To view a copy of this licence, visit http://creativecommons.org/licenses/by/4.0/.

\section{References}

1. Young WB. Transfer of strength and power training to sports performance. Int J Sports Physiol Perform. 2006;1(2):74-83.

2. Commission USCPS. National electronic injury surveillance system. Washington, DC: Directorate for Epidemiology, National Injury Information Clearinghouse; 1987.

3. Barker A, Lloyd RS, Buchheit M, Williams C, Oliver J. The BASES expert statement on trainability during childhood and adolescence. Sport Exerc Sci. 2014;41:22-3.

4. Lloyd RS, Faigenbaum AD, Stone MH, Oliver JL, Jeffreys I, Moody JA, et al. Position statement on youth resistance training: the 2014 International Consensus. Br J Sports Med. 2014;48(7):498-505.

5. Harries SK, Lubans DR, Callister R. Resistance training to improve power and sports performance in adolescent athletes: a systematic review and meta-analysis. J Sci Med Sport. 2012;15(6):532-40. https://doi.org/10.1016/j.jsams.2012.02.005.

6. Lesinski M, Prieske O, Granacher U. Effects and dose-response relationships of resistance training on physical performance in youth athletes: a systematic review and meta-analysis. Br J Sports Med. 2016;50(13):781-95. https://doi.org/10.1136/bjspo rts-2015-095497.

7. Morris RO, Jones B, Myers T, Lake J, Emmonds S, Clarke $\mathrm{ND}$, et al. Isometric midthigh pull characteristics in elite youth male soccer players: comparisons by age and maturity offset. J Strength Cond Res. 2018. https://doi.org/10.1519/jsc.00000 00000002673.

8. Brownlee TE, Murtagh CF, Naughton RJ, Whitworth-Turner CM, O'Boyle A, Morgans R, et al. Isometric maximal voluntary force evaluated using an isometric mid-thigh pull differentiates English Premier League youth soccer players from a maturity-matched control group. Sci Med Footb. 2018;2(3):209-15.

9. Bishop D. An applied research model for the sport sciences. Sports Med. 2008;38(3):253-63. https://doi.org/10.2165/00007 256-200838030-00005.

10. Côté $\mathbf{J}$. The influence of the family in the development of talent in sport. Sport Psychol. 1999;4:395-417.

11. Ford P, De Ste CM, Lloyd R, Meyers R, Moosavi M, Oliver J, et al. The long-term athlete development model: physiological evidence and application. J Sports Sci. 2011;29(4):389-402. https ://doi.org/10.1080/02640414.2010.536849.

12. Malina RM. Skeletal age and age verification in youth sport. Sports Med. 2011;41(11):925-47. 
13. Cobley S, Baker J, Wattie N, McKenna J. Annual age-grouping and athlete development. Sports Med. 2009;39(3):235-56.

14. Baxter-Jones AD, Eisenmann JC, Sherar LB. Controlling for maturation in pediatric exercise science. Pediatr Exerc Sci. 2005;17(1):18-30.

15. Tanner JM. Foetus into man: physical growth from conception to maturity. Cambridge: Harvard University Press; 1990.

16. Bayli I, Hamilton A. Long-term athlete development: trainability in childhood and adolescence: windows of opportunity, optional trainability. Victoria: Natl Coach Inst Br Columbia Adv Train Perform; 2004. p. 8.

17. Lloyd RS, Oliver JL. The youth physical development model: a new approach to long-term athletic development. Strength Cond J. 2012;34(3):61-72.

18. Moran J, Sandercock GR, Ramírez-Campillo R, Meylan C, Collison J, Parry DA. A meta-analysis of maturation-related variation in adolescent boy athletes' adaptations to short-term resistance training. J Sports Sci. 2017;35(11):1041-51.

19. Philippaerts RM, Vaeyens R, Janssens M, Van Renterghem B, Matthys D, Craen R, et al. The relationship between peak height velocity and physical performance in youth soccer players. J Sports Sci. 2006;24(3):221-30.

20. Murtagh CF, Brownlee TE, O'Boyle A, Morgans R, Drust B, Erskine RM. Importance of speed and power in elite youth soccer depends on maturation status. J Strength Cond Res. 2018;32(2):297-303. https://doi.org/10.1519/jsc.0000000000 002367.

21. Matthys S, Vaeyens R, Coelho-e-Silva M, Lenoir M, Philippaerts $\mathrm{R}$. The contribution of growth and maturation in the functional capacity and skill performance of male adolescent handball players. Int J Sports Med. 2012;33(07):543-9.

22. Beunen G, Malina RM. Growth and physical performance relative to the timing of the adolescent spurt. Exerc Sport Sci Rev. 1988;16(1):503-40.

23. O'Brien TD, Reeves ND, Baltzopoulos V, Jones DA, Maganaris $\mathrm{CN}$. Moment arms of the knee extensor mechanism in children and adults. J Anat. 2009;215(2):198-205.

24. Vingren JL, Kraemer WJ, Ratamess NA, Anderson JM, Volek JS, Maresh CM. Testosterone physiology in resistance exercise and training. Sports Med. 2010;40(12):1037-53.

25. Ramos E, Frontera W, Llopart A, Feliciano D. Muscle strength and hormonal levels in adolescents: gender related differences. Int J Sports Med. 1998;19(08):526-31.

26. Bhasin S, Storer TW, Berman N, Callegari C, Clevenger B, Phillips J, et al. The effects of supraphysiologic doses of testosterone on muscle size and strength in normal men. N Engl J Med. 1996;335(1):1-7.

27. Ferrando AA, Tipton KD, Doyle D, Phillips SM, Cortiella J, Wolfe RR. Testosterone injection stimulates net protein synthesis but not tissue amino acid transport. Am J Physiol. 1998;275(5):E864-E871871.

28. Bamman MM, Newcomer BR, Larson-Meyer DE, Weinsier RL, Hunter GR. Evaluation of the strength-size relationship in vivo using various muscle size indices. Med Sci Sports Exerc. 2000;32(7):1307-13.

29. Haff GG, Nimphius S. Training principles for power. Strength Cond J. 2012;34(6):2-12. https://doi.org/10.1519/SSC.0b013 e31826db467.

30. Vogler C, Bove K. Morphology of skeletal muscle in children. An assessment of normal growth and differentiation. Arch Pathol Lab Med. 1985;109(3):238-42.

31. Glenmark B, Hedberg G, Jansson E. Changes in muscle fibre type from adolescence to adulthood in women and men. A Acta Physiol Scand. 1992;146(2):251-9.

32. Gilliver SF, Degens H, Rittweger J, Sargeant AJ, Jones DA. Variation in the determinants of power of chemically skinned human muscle fibres. Exp Physiol. 2009;94(10):1070-8. https://doi. org/10.1113/expphysiol.2009.048314.

33. Malina RM, Bouchard C, Bar-Or O. Growth, maturation, and physical activity. Champaign: Human kinetics; 2004.

34. Degens H, Erskine RM, Morse CI. Disproportionate changes in skeletal muscle strength and size with resistance training and ageing. J Musculoskelet Neuronal Interact. 2009;9(3):123-9.

35. Close RI. Dynamic properties of mammalian skeletal muscles. Physiol Rev. 1972;52(1):129-97. https://doi.org/10.1152/physr ev.1972.52.1.129.

36. Jaric S, Markovic G. Leg muscles design: the maximum dynamic output hypothesis. Med Sci Sports Exerc. 2009;41(4):780-7.

37. Mersmann F, Bohm S, Schroll A, Boeth H, Duda G, Arampatzis A. Muscle and tendon adaptation in adolescent athletes: a longitudinal study. Scand J Med Sci Sports. 2017;27(1):75-82.

38. O'Brien TD, Reeves ND, Baltzopoulos V, Jones DA, Maganaris $\mathrm{CN}$. Muscle-tendon structure and dimensions in adults and children. J Anat. 2010;216(5):631-42.

39. Cunha GdS, Vaz MA, Herzog W, Geremia JM, Leites GT, Reischak-Oliveira Á. Maturity status effects on torque and muscle architecture of young soccer players. J Sports Sci. 2019;21:1-10.

40. Franchi MV, Fitze DP, Raiteri BJ, Hahn D, Spörri J. Ultrasoundderived biceps femoris long-head fascicle length: extrapolation pitfalls. Med Sci Sports Exerc. 2019;52(1):233-43.

41. Faigenbaum AD, Kraemer WJ, Blimkie CJ, Jeffreys I, Micheli LJ, Nitka M, et al. Youth resistance training: updated position statement paper from the national strength and conditioning association. J Strength Cond Res. 2009;23:S60-S79.

42. Meylan C, Cronin JB, Oliver J, Hopkins W, Contreras B. The effect of maturation on adaptations to strength training and detraining in 11- to 15-year-olds. Scand J Med Sci Sports. 2014;24(3):e156-e164164.

43. Rodríguez-Rosell D, Franco-Márquez F, Mora-Custodio R, González-Badillo JJ. Effect of high-speed strength training on physical performance in young soccer players of different ages. J Strength Cond Res. 2017;31(9):2498-508.

44. Behm DG, Young JD, Whitten JH, Reid JC, Quigley PJ, Low J, et al. Effectiveness of traditional strength vs. power training on muscle strength, power and speed with youth: a systematic review and meta-analysis. Front Physiol. 2017;8:423.

45. Behringer M, Heede AV, Matthews M, Mester J. Effects of strength training on motor performance skills in children and adolescents: a meta-analysis. Pediatr Exerc Sci. 2011;23(2):186-206.

46. Slimani M, Paravlic A, Granacher U. A meta-analysis to determine strength training related dose-response relationships for lowerlimb muscle power development in young athletes. Front Physiol. 2018;9:1155. https://doi.org/10.3389/fphys.2018.01155.

47. Legerlotz K, Marzilger R, Bohm S, Arampatzis A. Physiological adaptations following resistance training in youth athletes-a narrative review. Pediatr Exerc Sci. 2016;28(4):501-20.

48. Schwanbeck S, Chilibeck PD, Binsted G. A comparison of free weight squat to Smith machine squat using electromyography. J Strength Cond Res. 2009;23(9):2588-91.

49. Lloyd RS, Radnor JM, De Ste Croix MB, Cronin JB, Oliver JL. Changes in sprint and jump performances after traditional, plyometric, and combined resistance training in male youth pre- and post-peak height velocity. J Strength Cond Res. 2016;30(5):1239_ 47. https://doi.org/10.1519/jsc.0000000000001216.

50. Chaouachi A, Hammami R, Kaabi S, Chamari K, Drinkwater EJ, Behm DG. Olympic weightlifting and plyometric training with children provides similar or greater performance improvements than traditional resistance training. J Strength Cond Res. 2014;28(6):1483-96. https://doi.org/10.1519/jsc.0000000000 000305.

51. Chelly MS, Fathloun M, Cherif N, Amar MB, Tabka Z, Van Praagh E. Effects of a back squat training program on leg power, 
jump, and sprint performances in junior soccer players. J Strength Cond Res. 2009;23(8):2241-9.

52. Schmidtbleicher D. Training for power events. Oxford: Blackwell Science Ltd; 2004.

53. Wing CE, Turner AN, Bishop CJ. The importance of strength and power on key performance indicators in elite youth soccer. J Strength Cond Res. 2018. https://doi.org/10.1519/JSC.00000 00000002446.

54. Comfort P, Stewart A, Bloom L, Clarkson B. Relationships between strength, sprint, and jump performance in well-trained youth soccer players. J Strength Cond Res. 2014;28(1):173-7. https://doi.org/10.1519/JSC.0b013e318291b8c7.

55. Lloyd RS, Oliver J. Strength and conditioning for young athletes. Abingdon: Taylor \& Francis; 2013.

56. Couppe C, Kongsgaard M, Aagaard P, Vinther A, Boesen M, Kjaer M, et al. Differences in tendon properties in elite badminton players with or without patellar tendinopathy. Scand J Med Sci Sports. 2013;23(2):e89-95. https://doi.org/10.1111/sms.12023.

57. Murtagh CF, Naughton RJ, McRobert AP, O'Boyle A, Morgans R, Drust B, et al. A coding system to quantify powerful actions in soccer match play: a pilot study. Res Q Exerc Sport. 2019;90(2):234-43.

58. Hunter JP, Marshall RN, McNair PJ. Relationships between ground reaction force impulse and kinematics of sprint-running acceleration. J Appl Biomech. 2005;21(1):31-433.

59. Cronin JB, Hansen KT. Strength and power predictors of sports speed. J Strength Cond Res. 2005;19(2):349-57. https://doi. org/10.1519/14323.1.

60. Seitz LB, Reyes A, Tran TT, de Villarreal ES, Haff GG. Increases in lower-body strength transfer positively to sprint performance: a systematic review with meta-analysis. Sports Med. 2014;44(12):1693-702. https://doi.org/10.1007/s4027 9-014-0227-1.

61. Wisloff U, Castagna C, Helgerud J, Jones R, Hoff J. Strong correlation of maximal squat strength with sprint performance and vertical jump height in elite soccer players. Br J Sports Med. 2004;38(3):285-8

62. Keiner M, Sander A, Wirth K, Caruso O, Immesberger P, Zawieja $M$. Strength performance in youth: trainability of adolescents and children in the back and front squats. J Strength Cond Res. 2013;27(2):357-62. https://doi.org/10.1519/JSC.0b013e3182 $576 \mathrm{fbf}$.

63. Pallarés JG, Cava AM, Courel-Ibáñez J, González-Badillo JJ, Morán-Navarro R. Full squat produces greater neuromuscular and functional adaptations and lower pain than partial squats after prolonged resistance training. Eur J Sport Sci. 2019;20(1):115-24.

64. Haff GG. Quantifying workloads in resistance training: a brief review. Strength Cond J. 2010;10:31-40.

65. Lloyd RS, Oliver JL, Faigenbaum AD, Myer GD, Croix MBDS. Chronological age vs. biological maturation: implications for exercise programming in youth. J Strength Cond Res. 2014;28(5):1454-64.

66. Speirs DE, Bennett MA, Finn CV, Turner AP. Unilateral vs. bilateral squat training for strength, sprints, and agility in Academy Rugby Players. J Strength Cond Res. 2016;30(2):386-92. https:// doi.org/10.1519/jsc.0000000000001096.

67. Hammami M, Negra Y, Billaut F, Hermassi S, Shephard RJ, Chelly MS. Effects of lower-limb strength training on agility, repeated sprinting with changes of direction, leg peak power, and neuromuscular adaptations of soccer players. J Strength Cond Res. 2018;32(1):37-47. https://doi.org/10.1519/jsc.000000000000181 3.

68. Styles WJ, Matthews MJ, Comfort P. Effects of strength training on squat and sprint performance in soccer players. J Strength Cond Res. 2016;30(6):1534-9. https://doi.org/10.1519/jsc.00000 00000001243
69. Peterson MD, Rhea MR, Alvar BA. Maximizing strength development in athletes: a meta-analysis to determine the dose-response relationship. J Strength Cond Res. 2004;18(2):377-82.

70. Sander A, Keiner M, Wirth K, Schmidtbleicher D. Influence of a 2-year strength training programme on power performance in elite youth soccer players. Eur J Sport Sci. 2013;13(5):445-51. https:// doi.org/10.1080/17461391.2012.742572.

71. Plisk SS, Stone MH. Periodization strategies. Strength Cond J. 2003;25(6):19-37.

72. Hori N, Newton R, Stone M. Weightlifting exercises enhance athletic performance that requires high-load speed strength. Strength Cond J. 2005;24(4):50-5.

73. Garhammer J, Gregor R. Propulsion forces as a function of intensity for weightlifting and vertical jumping. J Appl Sport Sci Res. 1992;6(3):129-34.

74. Canavan PK, Garrett GE, Armstrong LE. Kinematic and kinetic relationships between an olympic-style lift and the vertical jump. J Strength Cond Res. 1996;10(2):127-30.

75. Channell BT, Barfield J. Effect of Olympic and traditional resistance training on vertical jump improvement in high school boys. J Strength Cond Res. 2008;22(5):1522-7.

76. Arabatzi F, Kellis E. Olympic weightlifting training causes different knee muscle-coactivation adaptations compared with traditional weight training. J Strength Cond Res. 2012;26(8):2192201. https://doi.org/10.1519/JSC.0b013e31823b087a.

77. Baker D, Newton RU. Acute effect on power output of alternating an agonist and antagonist muscle exercise during complex training. J Strength Cond Res. 2005;19(1):202-5. https://doi. org/10.1519/1533-4287(2005)19<202:Aeopoo>2.0.Co;2.

78. Negra Y, Chaabene H, Hammami M, Hachana Y, Granacher U. Effects of high-velocity resistance training on athletic performance in prepuberal male soccer athletes. J Strength Cond Res. 2016;30(12):3290-7. https://doi.org/10.1519/jsc.0000000000 001433.

79. Cormie P, McCaulley GO, Triplett NT, McBride JM. Optimal loading for maximal power output during lower-body resistance exercises. Med Sci Sports Exerc. 2007;39(2):340-9. https://doi. org/10.1249/01.mss.0000246993.71599.bf.

80. Nuzzo JL, McBride JM, Cormie P, McCaulley GO. Relationship between countermovement jump performance and multijoint isometric and dynamic tests of strength. J Strength Cond Res. 2008;22(3):699-707.

81. Gonzalez-Badillo JJ, Pareja-Blanco F, Rodriguez-Rosell D, AbadHerencia JL, Del Ojo-Lopez JJ, Sanchez-Medina L. Effects of velocity-based resistance training on young soccer players of different ages. J Strength Cond Res. 2015;29(5):1329-38. https://doi. org/10.1519/jsc.0000000000000764.

82. Ebben WP. Complex training: a brief review. J Sports Sci Med. 2002;1(2):42.

83. Bauer P, Uebellacker F, Mitter B, Aigner AJ, Hasenoehrl T, Ristl $\mathrm{R}$, et al. Combining higher-load and lower-load resistance training exercises: a systematic review and meta-analysis of findings from complex training studies. J Sci Med Sport. 2019;22(7):838-51.

84. Radnor JM, Lloyd RS, Oliver JL. Individual response to different forms of resistance training in school-aged boys. J Strength Cond Res. 2017;31(3):787-97. https://doi.org/10.1519/jsc.0000000000 001527.

85. Pichardo AW, Oliver JL, Harrison CB, Maulder PS, Lloyd RS, Kandoi R. Effects of combined resistance training and weightlifting on motor skill performance of adolescent male athletes. J Strength Cond Res. 2019;33(12):3226-355. 